\title{
Étude et gestion d'une importante éclosion communautaire d'oreillons parmi les jeunes adultes de Toronto, Canada, de janvier 2017 à février 2018
}

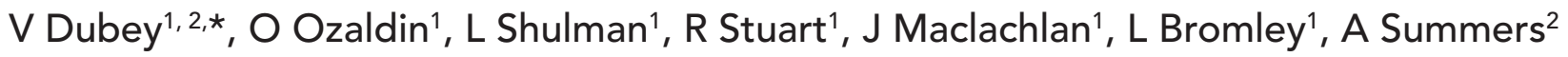

\section{Résumé}

Contexte : En 2017, une éclosion d'oreillons a été mise au jour au sein d'une cohorte de personnes de 18 à 34 ans à Toronto au Canada.

Objectif : Décrire une importante éclosion communautaire d'oreillons chez de jeunes adultes situés dans un centre urbain du mois de janvier 2017 au mois de février 2018.

Méthodologie : Une vaste gamme d'interventions ont été mises en place afin d'atteindre le public cible, dont la gestion des personnes atteintes et des contacts ; des cliniques de vaccination en milieu scolaire et au cabinet du médecin ; des exclusions scolaires ; l'inspection des bars ; des stratégies de communication traditionnelles (y compris l'avertissement des fournisseurs de soins de santé et des affiches) et de nouvelles stratégies de communication (dont trois campagnes séquentielles sur les réseaux sociaux).

Résultats : Au total, 143 cas d'oreillons ont été répertoriés. Bien que l'âge des personnes atteintes était répartie entre trois ans et 72 ans, la plupart (76\%) étaient âgés de 18 à 34 ans, et plusieurs d'entre elles avaient fréquenté des bars et des établissements alimentaires du centre-ville de Toronto. $84 \%(n=120)$ des cas étaient d'origine communautaire. Seulement $16 \%(n=23)$ des cas déclarés résultaient d'une exposition dans les écoles et les institutions scolaires post-secondaires. $39 \%(n=56)$ des cas ignoraient leurs antécédents de vaccination; $34 \%(n=49)$ étaient soit non vaccinés, soit partiellement vaccinés par une seule dose du vaccin antirougeoleux, antiourlien et antirubéoleux et $27 \%(n=38)$ avaient reçu les deux doses recommandées du vaccin antiourlien. L'absence d'un registre a la détermination du statut vaccinal. La vaccination était recommandée lorsque les sujets avaient reçu moins de deux doses du vaccin ou que leur statut vaccinal était inconnu. Une campagne sur les réseaux sociaux, mettant l'accent sur les risques des activités sociales en l'absence d'une protection contre les oreillons, a engendré plus de 500000 réactions à des messages et des publicités publiés sur Facebook et Twitter de même qu'un impressionnant taux de mobilisation allant de $1 \%$ à $10 \%$.

Conclusion : Il s'agit de la plus importante éclosion d'oreillons à Toronto en vingt ans. Chez les jeunes adultes, les campagnes de communication traditionnelles et sur les réseaux sociaux peuvent contribuer au contrôle des éclosions communautaires d'oreillons. Encourager la prise vaccinale est une bonne chose mais, sans la présence d'un registre d'immunisation, il est difficile d'évaluer la couverture vaccinale chez les personnes adultes. Les cohortes vulnérables de jeunes adultes qui n'ont pas été immunisés de façon adéquate sont à risque de futures éclosions. Étant donné que presque $30 \%$ des personnes atteintes avaient déjà reçu les deux doses recommandées d'un vaccin antiourlien, il est possible que deux doses ne soient pas suffisantes pour offrir une protection complète.
Citation proposée : Dubey V, Ozaldin O, Shulman L, Stuart R, Maclachlan J, Bromley L, Summers A. Étude et gestion d'une importante éclosion communautaire d'oreillons parmi les jeunes adultes de Toronto, Canada, de janvier 2017 à février 2018.

Relevé des maladies transmissibles au Canada 2018;44(12):351-9. https://doi.org/10.14745/ccdr.v44i12a01f RRO, Toronto

Affiliations

${ }^{1}$ Bureau de santé publique de Toronto, Ontario

2 École de santé publique Dalla Lana, Université de Toronto, Toronto (Ontario)

*Correspondance :

vinita.dubey@toronto.ca 


\section{Introduction}

De janvier 2017 à février 2018, Toronto a vécu sa plus grande éclosion d'oreillons des 20 dernières années, avec 143 cas. Le plus grand centre urbain du Canada, Toronto, compte une population de 2,7 millions de personnes. Elle présente en moyenne cinq cas d'oreillons par année, largement associés aux voyages. La dernière grande éclosion était survenue en 2009 avec 33 cas.

Les oreillons sont une infection virale causée par un paramyxovirus qui peut engendrer des symptômes de fièvre, des malaises, des maux de tête, la myalgie et la parotidite. L'orchite est une complication fréquente observée chez les hommes postpubertaires. Bien qu'un tiers des cas ne présente que des symptômes bénins, les complications peuvent comprendre la méningite, la pancréatite, la myocardite et la surdité. Les symptômes sont souvent plus graves chez les adultes que chez les enfants. La période d'incubation est de 12 à 25 jours et le virus se propage au moyen de gouttelettes et de contacts directs avec la salive ou des gouttelettes respiratoires, de sept jours avant jusqu'à cinq jours après l'apparition des symptômes. La contagiosité est similaire à cette de la grippe (1-3).

On présume généralement que les adultes nés avant 1970 ont acquis une immunité naturelle aux oreillons. En Ontario, une seule dose du vaccin antirougeoleux, antiourlien et antirubéoleux (rougeole-oreillons-rubéole [ROR]) était administrée entre 1975 et 1996. En 1996, une deuxième dose de ce vaccin a été ajoutée au calendrier de vaccination et une dose unique du vaccin monovalent de la rougeole était offerte à tous les élèves de 4 à 18 ans (nés entre 1978 et 1992) (4,5). Les taux de couverture pour deux doses de vaccin contenant le virus des oreillons chez les enfants d'âge scolaire ont été constants et d'environ $90 \%$ au cours des dix dernières années dans les écoles de Toronto $(6,7)$.

Ce plan de vaccination a laissé pour compte une cohorte de personnes nées après 1970 et avant 1992 qui n'ont reçu qu'une seule dose de vaccin contenant le virus des oreillons. Le Comité consultatif national de l'immunisation a recommandé qu'au cours d'une éclosion d'oreillons, cette cohorte reçoive une dose de vaccin contenant le virus des oreillons. Cependant cette cohorte est reconnue comme étant difficile à joindre (8).

Les registres de vaccination sont des outils importants pour documenter et améliorer la couverture vaccinale. Lorsque surgissent des éclosions de maladies évitables par la vaccination, un registre peut confirmer les antécédents d'immunisation et permet d'évaluer rapidement les personnes vulnérables qui nécessitent un vaccin parmi une population donnée.

L'objectif du présent article est de décrire l'importante éclosion communautaire d'oreillons récemment survenue à Toronto ainsi que les approches novatrices de communication et de contrôle de l'éclosion qui ont été mises en place à l'aide des réseaux sociaux et d'affiches.

\section{Détection de l'éclosion}

L'éclosion a commencé en janvier 2017 lorsque deux jeunes adultes de la même famille ayant respectivement 18 et 20 ans ont été signalés à la Santé publique de Toronto. Ils étaient atteints d'une d'infection aux oreillons, confirmée en laboratoire. Ils présentaient tous deux des symptômes de fièvre, de la fatigue et une parotidite. On a déterminé que l'infection avait probablement été contractée à la mi-janvier au cours d'une fête privée dans une maison de Guelph en Ontario (une petite ville située à environ 100 kilomètres au sud-ouest de Toronto). Des cas ont aussi été détectés ailleurs en Ontario en lien avec I'exposition à cette fête privée. D'autres cas ont été détectés chez de jeunes adultes ayant des liens avec des bars et des établissements alimentaires du centre-ville de Toronto et n'ayant pas de liens clairement définis avec la fête privée de Guelph, des voyages ou d'autres personnes atteintes. Une éclosion d'oreillons a été déclarée par la Ville de Toronto le 30 janvier 2017.

\section{Intervention contre l'éclosion}

\section{Définitions des cas et enquêtes à leur sujet}

Les définitions des cas de l'éclosion sont résumées à l'annexe 1. Tous les échantillons de laboratoires testés positifs pour les oreillons en Ontario ont été directement signalés au bureau de santé publique local pour un suivi selon le Protocole concernant les maladies infectieuses des Normes de santé publique de I'Ontario (9). Le personnel de la santé publique a par la suite interrogé l'ensemble des personnes porteuses des oreillons en utilisant un outil d'enquête de cas du système d'information en santé publique intégré qui a été adapté tout spécialement à la présente éclosion. On a demandé aux clients de fournir des renseignements portant sur leurs antécédents de vaccination, leurs symptômes, leur emploi, leur fréquentation des milieux de soins de santé et de milieux scolaires, leurs facteurs de risques médicaux et sociaux, et les sites potentiels d'exposition pour I'acquisition et la transmission. Dès le début de l'éclosion, il était évident que les clients $n$ 'étaient pas communicatifs dans leurs réponses à certaines questions, particulièrement les questions associées aux détails sur les contacts et les éventuels sites d'exposition.

Le laboratoire de Santé publique Ontario a transmis les échantillons au Laboratoire national de microbiologie à des fins de génotypage. En raison des retards subis dans la transmission des résultats, le génotypage n'a pas été introduit dans les définitions de cas.

Des analyses descriptives visant à évaluer les données démographiques et géographiques, le statut vaccinal, le génotype et les symptômes associés à chacun des cas ont été réalisées. L'analyse des réseaux sociaux a été envisagée très tôt au début de l'éclosion, mais, comme les personnes atteintes n'étaient pas très communicatives concernant leurs expositions 
et leurs réseaux sociaux, les renseignements n'étaient pas suffisamment nombreux pour poursuivre cette analyse.

\section{Gestion des personnes atteintes et des contacts}

Les cas d'oreillons ont été gérés de manière traditionnelle $(3,9)$. Les personnes atteintes devaient s'isoler et ne pas fréquenter leur milieu de travail, l'école, les rassemblements sociaux, ni les établissements de soins de santé pendant la période de contagion (cinq jours après l'apparition des symptômes). Des entrevues ont été réalisées auprès de ces personnes afin de cerner les sites potentiels de contraction et de transmission au cours des périodes d'incubation et de contagion. La gestion des contacts employée lors de l'éclosion est résumée à l'annexe 2.

\section{Avertissement des fournisseurs de soins de santé}

En Ontario, la majorité des séances de vaccination sont tenues par des pourvoyeurs de soins de santé primaires. Un grand nombre de messages ont été envoyés aux fournisseurs de vaccins afin de les informer sur le statut de l'éclosion, de leur fournir des instructions portant sur la manière de la diagnostiquer et de procéder à l'examen des oreillons ainsi que les encourager à vacciner tous leurs patients âgés de 18 à 35 ans. Comme à Toronto les vaccins antiourliens sont commandés par les fournisseurs de soins de santé et expédiés du Service d'approvisionnement médico-pharmaceutique du gouvernement de l'Ontario, la base de données d'inventaire des vaccins Panorama a servi à déterminer le nombre de vaccins antiourliens qui ont été commandés et expédiés entre les mois de mars et d'août 2017, comparativement à la même période en 2018 (après l'éclosion).

\section{Exclusion scolaire obligatoire des élèves susceptibles d'avoir été en contact avec des personnes infectées}

La Loi sur l'immunisation des élèves de l'Ontario exige que tous les élèves soient vaccinés contre certaines maladies ou qu'ils aient demandé une exemption pour des raisons médicales, philosophiques ou religieuses (10). Dans le contexte d'une éclosion, les responsables de la santé publique peuvent exclure les élèves dont le statut vaccinal n'est pas à jour ou qui n'ont pas de preuve de leur vaccination. Dans les écoles où un cas a été déclaré, une attention particulière a été portée à la mise à jour des dossiers de vaccination et à la vaccination des élèves dont le statut d'immunisation ne comprenait pas deux doses de vaccins antiourliens. Dans une école secondaire où deux cas possibles de transmission ont été signalés, les élèves dont le statut vaccinal n'était pas à jour ou qui n'avaient pas été vaccinés contre les oreillons ont été exclus de l'école jusqu'à ce qu'ils puissent démontrer leur vaccination. On a tenu dans les écoles des cliniques de vaccination afin de mettre à jour les dossiers de vaccination et de rapidement vacciner les membres du personnel et les élèves. De nouvelles transmissions ne se sont pas produites dans les écoles primaires et secondaires.

\section{Inspections des bars}

Dans la phase initiale de l'éclosion, des bars qui avaient été fréquentés par des personnes atteintes des oreillons au moment de leur période de contagion ont été inspectés. Les inspections se sont concentrées sur la prévention des infections potentielles et le manque de contrôle qui pourrait expliquer la transmission, comme un nettoyage et une désinfection inadéquats de la vaisselle et des verres. Une lettre et une fiche d'information sur les oreillons ont été élaborées et remises aux propriétaires des bars.

\section{Stratégie de communication}

Une stratégie de communication a été élaborée dans le but de cibler les jeunes adultes qui fréquentent souvent les bars situés à l'ouest du centre-ville de Toronto. Les messages visaient à sensibiliser le public cible à l'infection par les oreillons et à sa transmission, et faisaient la promotion de la vaccination. Plus de 70 entrevues ont été accordées dans de multiples réseaux et émissions d'informations. Des lettres et des affiches ont été créées et distribuées à différents groupes afin de rejoindre le public cible des jeunes adultes (figure 1). Les centres communautaires ont été identifiés à l'aide des listes internes de la ville et les gymnases ont été répertoriés à partir des listes disponibles sur Internet. L'ensemble des institutions post-secondaires de Toronto ont été repérées et de la documentation leur a été envoyée au mois août 2017, avant la semaine d'initiation et le début des classes.

Puisque plusieurs personnes atteintes ont nommé des bars et des restaurants du centre-ville comme lieux possibles d'exposition tôt lors de l'éclosion, plus de 4000 lettres ont été postées à des établissements du centre-ville. Plusieurs employés de ces bars ayant été répertoriés comme des cas avérés, des affiches destinées à ces personnes ont aussi été créées et distribuées au plus fort de l'éclosion.

Une page Web portant sur l'éclosion a été créée et mise à jour régulièrement. Elle figurait un décompte des nouveaux cas ainsi que des messages de prévention.

\section{Stratégie sur les réseaux sociaux}

Trois campagnes ont été lancées au cours de l'éclosion sur Facebook et Twitter. Pendant la première vague, une campagne sur les réseaux sociaux s'est déroulée de février à avril 2017, ciblant les jeunes socialement actifs à l'ouest du centre-ville de Toronto. Le but était de leur faire prendre conscience de l'éclosion à Toronto et de les encourager à vérifier leur carnet de vaccination ou de consulter leur médecin afin de s'assurer que leur immunisation était à jour. On a conçu des images créatives reflétant leur style, leurs attitudes et leurs comportements en ligne (figure 1). Voici un exemple des messages envoyés sur les réseaux sociaux pendant l'éclosion d'oreillons de Toronto : 
- Répandez l'amour, pas les oreillons. N'échangez pas vos boissons, vos ustensiles, votre nourriture ou vos bouteilles d'eau

- Votre style est au goût du jour. Et vos vaccins? Assurez-vous d'être protégé contre les oreillons

Figure 1 : Exemple d'affiche et d'image des réseaux sociaux utilisées dans le cadre de l'éclosion d'oreillons de Toronto, 2017-2018

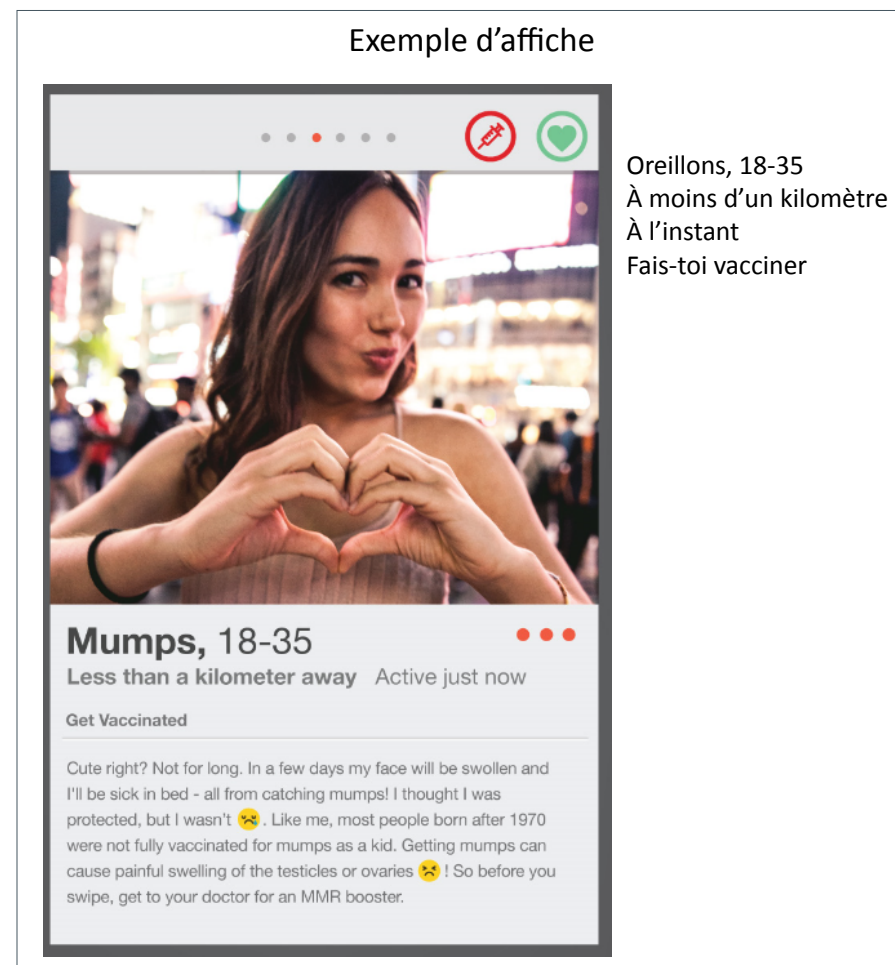

Jolie, n'est-ce pas? Pas pour longtemps! Dans quelques jours, mon visage sera enflé et je serai au lit et très malade - tout cela parce que j’ai attrapé les oreillons! Je pensais être protégée, mais je ne l'étais pas :'(. Comme moi, la plupart des personnes nées après 1970 n'ont pas été complètement immunisées contre les oreillons quand elles étaient enfants. Attraper les oreillons peut causer une enflure douloureuse des testicules ou des ovaires :(! Donc, avant de swiper, allez consulter votre médecin pour obtenir un rappel du vaccin.

\section{Échantillon d'une image des réseaux sociaux}

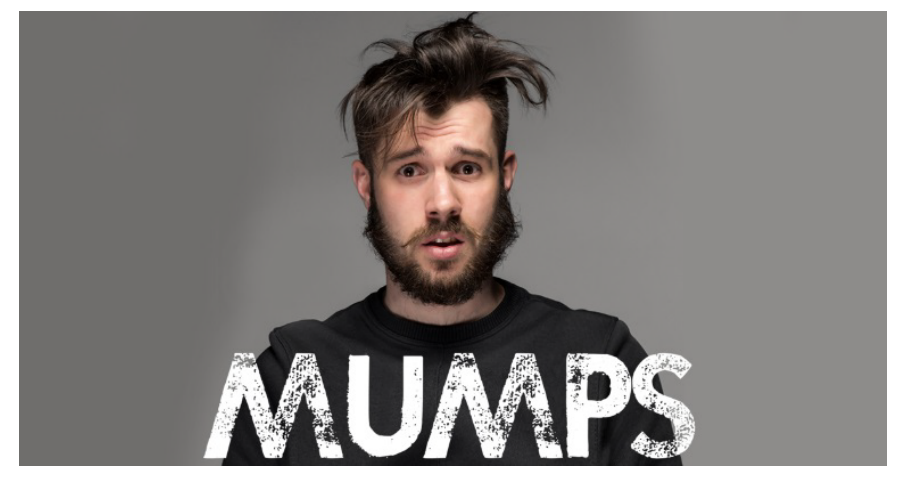

${ }^{a}$ Traduction de la première image : Oreillons, 18-35. À l'instant. Fais-toi vacciner. Jolie, n'est-ce pas? Pas pour longtemps! Dans quelques jours, mon visage sera enflé et je serai au lit et très malade - tout cela parce que j'ai attrapé les oreillons! Je pensais être protégée, mais je ne l'étais pas. Comme moi, la plupart des personnes nées après 1970 n'ont pas été complètement vaccinées contre les oreillons quand elles étaient enfants. Attraper les oreillons peut causer une valure douloureuse des testicules ou des ovaires! Donc, avant de l'attraper, allez consulter votre inces ou des ovaires! Donc, avant de l'attraper, allez consulter votre médecin pour obtenir un rappel du vaccin

${ }^{B}$ Traduction de la deuxième image : Oreillons
- Le mot mumps est plus qu'une drôle d'expression en anglais - les oreillons sont en hausse à Toronto

- Attrapez l'amour cet été, pas les oreillons. Parlez à votre médecin du vaccin ROR

La deuxième campagne de réseaux sociaux s'est déroulée de juillet à septembre 2017. Elle était accompagnée d'une conception créative améliorée et d'un appel à l'action plus dynamique. Puisqu'il devenait clair que l'éclosion ne tirait pas à sa fin et qu'une augmentation de l'immunité collective était essentielle, on est passé de messages de type «apprenez-en davantage» à «obtenez le vaccin». On a retravaillé les images et les messages afin de les associer aux activités estivales qui pourraient aggraver la transmission.

À la suite d'une autre vague de cas à l'automne, une campagne se concentrant sur des images et des messages ayant les fêtes et I'hiver pour thématique a été lancée en décembre. Le message principal était d'obtenir le vaccin.

\section{Résultats}

\section{Description de l'éclosion}

Au total, 143 cas d'oreillons ont été répertoriés du 1 er janvier 2017 au 26 février 2018. L'éclosion a présenté un sommet initial au début mars 2017 et, dès le mois de juin 2017, le nombre de cas avait sensiblement décliné (figure 2). Un second sommet a commencé à la fin août et a perduré tout au long de l'automne avant de décliner pour le reste de 2017. On a déclaré terminée l'éclosion d'oreillons le 26 février 2018, soit 50 jours (deux périodes d'incubation) après l'apparition du dernier cas.

Soixante-seize pourcent des personnes atteintes avaient entre 18 et 34 ans. La moyenne d'âge des cas de l'éclosion était de 28 ans (fourchette s'étendant entre 3 et 72 ans). Les hommes

Figure 2 : Courbe épidémique des cas d'oreillons confirmés par semaine à Toronto du 1 ${ }^{\text {er }}$ janvier 2017 au 26 février 2018

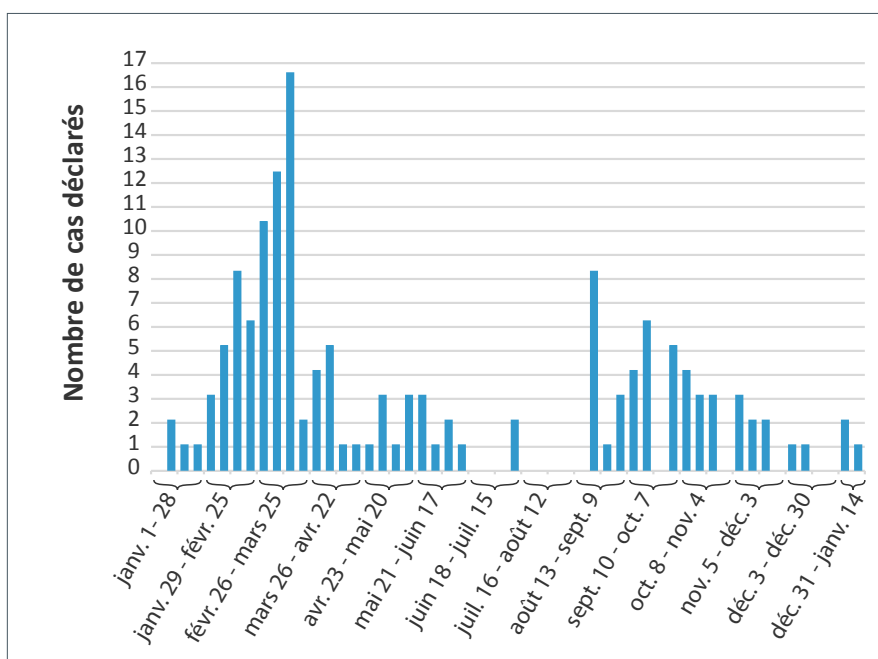

Semaine du déclenchement 
et les femmes étaient représentés assez équitablement (55\% d'hommes). La plupart des cas (84\%) avaient contracté la maladie hors établissement et seulement $16 \%$ d'entre eux représentaient soit un employé ou un élève d'un établissement d'études primaires, secondaires ou post-secondaires; une transmission soutenue ne s'est pas produite dans ces établissements (tableau 1).

Tableau 1 : Résumé descriptif des cas d'oreillons de Toronto du $1^{\text {er }}$ janvier 2017 au 26 février 2018

\begin{tabular}{|c|c|c|}
\hline \multirow{2}{*}{$\begin{array}{l}\text { Caractéristiques } \\
\text { descriptives }\end{array}$} & \multicolumn{2}{|c|}{ Cas déclarés (\%) } \\
\hline & $n$ & (\%) \\
\hline Nombre de cas & 143 & 100 \\
\hline \multicolumn{3}{|l|}{$\hat{A} g e^{a}$ (ans) } \\
\hline $0-5$ & 1 & 1 \\
\hline 6-11 & 0 & 0 \\
\hline $12-17$ & 9 & 6 \\
\hline $18-25$ & 44 & 31 \\
\hline $26-34$ & 65 & 45 \\
\hline $35-49$ & 21 & 15 \\
\hline $50-64$ & 2 & 1 \\
\hline 65 ou plus & 1 & 1 \\
\hline \multicolumn{3}{|l|}{ Sexe } \\
\hline Hommes & 79 & 55 \\
\hline Femmes & 64 & 45 \\
\hline \multicolumn{3}{|c|}{ Expositions en milieu scolaire ${ }^{b}$} \\
\hline Oui & 11 & 8 \\
\hline Non & 132 & 92 \\
\hline \multicolumn{3}{|l|}{ Expositions dans des bars ${ }^{b}$} \\
\hline Oui & 70 & 49 \\
\hline Non & 73 & 51 \\
\hline \multicolumn{3}{|c|}{ Expositions en établissement scolaire post-secondaire ${ }^{b}$} \\
\hline Oui & 11 & 8 \\
\hline Non & 132 & 92 \\
\hline \multicolumn{3}{|l|}{ Statut vaccinal } \\
\hline Vacciné & 38 & 27 \\
\hline Non vacciné & 16 & 11 \\
\hline Partiellement vacciné & 33 & 23 \\
\hline Inconnu & 56 & 39 \\
\hline
\end{tabular}

Abréviation : $\mathrm{n}$, nombre

a Au cours de cette éclosion, l'âge moyen était de 28 ans. L'âge variait de trois à 72 ans

${ }^{b}$ Les personnes infectées peuvent avoir déclaré plus d'un site d'exposition. Les taux de couverture pour deux doses de vaccin contenant le virus des oreillons chez les enfants d'âge scolaire sont demeurés autour de $90 \%$ au cours des dix dernières années dans les écoles de Toronto $(6,7)$

La parotidite était le symptôme le plus fréquemment cité, avec $97 \%(n=139)$ des cas. Les complications graves ont été rares parmi les personnes infectées : seules deux personnes parmi les 143 cas ont dû consulter le service des urgences en raison de leurs symptômes et une seule d'entre elles a été hospitalisée. L'orchite a été mentionnée par $23 \%(n=18)$ des hommes infectés.

La plupart des cas (73\%) étaient soit non vaccinés (11\%), étaient partiellement vaccinés par une seule dose du vaccin RRO (23\%) ou présentaient des antécédents d'immunisation inconnus (39\%). Seulement $27 \%$ des personnes infectées avaient des antécédents d'immunisation connus présentant deux doses du vaccin RRO. Cinq personnes (3\%) nées avant 1970, dont on présumait qu'elles avaient acquis une immunité en raison de leur âge, avaient aussi attrapé les oreillons.

La plupart des cas répertoriés au cours de l'éclosion avaient été contaminés localement (93\%). Sur les 139 cas dont le génotype a été examiné, la majorité $(n=115)$ présentait le génotype $G$. On a aussi détecté un cas de génotype $C$ et un cas de génotype $K$, tous deux associés à des voyages. Les cas associés aux voyages ont été compris dans l'éclosion, car ils étaient présents à Toronto pendant au moins une partie de leur période d'incubation et les renseignements concernant le génotype n'étaient pas inclus dans les définitions de cas. Le reste des cas $(n=22)$ étaient indéterminés.

Initialement, la plupart des nouveaux cas n'étaient pas clairement associés entre eux ou à des établissements communs. Cependant, l'évaluation épidémiologique a permis de déterminer que des cas s'étaient produits dans l'ouest du centre-ville de Toronto et que des expositions fréquentes dans des douzaines de bars et de restaurants de ce quartier ont été signalées, soit par un client ou un employé de ces établissements. À mesure que l'éclosion progressait, la majorité des cas ne signalaient plus seulement des expositions dans les bars ou dans l'ouest du centre-ville de Toronto, mais aussi une transmission communautaire généralisée à l'échelle de la ville était évidente.

\section{Ordonnances de vaccin contenant le virus des oreillons par les fournisseurs de soins primaires}

Au cours de la période allant de mars à août 2017, le Service d'approvisionnement médico-pharmaceutique du gouvernement de l'Ontario a acheminé 78680 doses de vaccin contenant le virus des oreillons à la demande des fournisseurs de soins de santé de Toronto, ce qui représentait une moyenne de 13113 doses par mois. Au cours de la période correspondante en 2018, seules 66509 doses du vaccin ont été acheminées, soit une moyenne de 11085 par mois. On voit à ces chiffres que 12000 doses de plus ont été acheminées au sommet de l'éclosion en 2017, comparativement à la même période l'année suivante. 


\section{Performance des campagnes sur les réseaux sociaux}

Le résultat des messages envoyés sur les réseaux sociaux a dépassé les attentes. Au cours de la première campagne se déroulant de février à avril 2017, au moment du sommet de la première vague de l'éclosion, il y a eu plus de 360000 expositions à des messages et des annonces de Facebook et de Twitter, et plus de 14000 engagements. Le taux d'engagement sur Twitter a atteint $10 \%$, comparativement au compte du Bureau de santé publique de Toronto, qui totalisait en moyenne près de $1 \%$. Pour la campagne de l'été 2017, le taux d'engagement sur Facebook et Twitter demeurait encore élevé, à $1 \%$, et les comptes ont atteint 50000 expositions additionnelles. La troisième campagne de décembre a encore maintenu un taux d'engagement élevé, de $2 \%$, ce qui représentait presque 120000 expositions.

On a évalué la réception de la campagne en étudiant les commentaires et les réactions aux messages de la campagne. Dans l'ensemble, le nombre de réponses positives (J'aime, $\mathrm{J}$ 'adore et rires) dépassait largement le nombre de réponses négatives. Les commentateurs appréciaient le ton humoristique et prenaient note de l'importance de la vaccination. Comme on s'y attendait, des commentaires anti-vaccins étaient aussi présents.

La page Web de l'enquête sur l'éclosion d'oreillons a connu une augmentation importante de son trafic en ligne, qui est passé de 161 consultations par jour en janvier 2017 à 13698 consultations de février à avril 2017, au sommet de l'éclosion. Les consultations Web augmentaient lorsque la couverture médiatique était plus grande et était retransmise par des personnes influentes ou des publicités sur Facebook.

\section{Discussion}

Cette éclosion communautaire constituée en grande partie de jeunes adultes de 18 à 34 ans est apparue dans une zone géographique distincte dans les bars et les restaurants situés dans l'ouest du centre-ville de Toronto puis s'est étendue à l'ensemble de la ville.

Même si certains jeunes adultes faisaient partie de la cohorte née après 1970 et avant 1992 qui n'avait reçu dans l'enfance qu'une seule dose du vaccin antiourlien, $50 \%$ des personnes atteintes présentaient un statut vaccinal inconnu ou n'étaient pas vaccinées. Cinq personnes (3\%) nées avant 1970, dont on présumait qu'elles avaient acquis une immunité en raison de leur âge, ont aussi attrapé les oreillons. Sans registre vaccinal, il est difficile de déterminer le nombre de personnes dont le statut vaccinal est inconnu, mais qui étaient en fait immunisées. Un registre permettrait aussi de calculer le temps écoulé depuis la dernière vaccination, ce qui pourrait être un important indicateur de l'immunité aux oreillons acquise par la vaccination sur le lieu d'une éclosion (11).
Cette éclosion a présenté des problèmes particuliers en ce qui a trait à la recherche des contacts et à la transmission des messages de santé publique, d'autant plus qu'elle n'a pas commencé dans un établissement ou chez un groupe de personnes bien défini. Il était difficile de joindre les clients par les moyens traditionnels que sont les appels téléphoniques et les lettres. Comme plusieurs étaient hésitants à fournir les coordonnées de proches présentant des symptômes (amis, collègues ou partenaires sexuels occasionnels), la responsabilité de les informer reposait sur les personnes atteintes. Certaines personnes atteintes travaillaient dans des établissements alimentaires et hésitaient à fournir leurs coordonnées au travail, car elles étaient inquiètes de la publicité négative pouvant en découler pour l'établissement en plus du risque de perdre leur emploi.

La plupart des jeunes adultes préfèrent entrer en communication et recevoir des renseignements au moyen de messages textes et sur les réseaux sociaux plutôt que par des méthodes plus traditionnelles comme les journaux ou des lettres. Les plateformes comme Twitter et Facebook ont été choisies comme des canaux idéaux permettant de rejoindre rapidement et efficacement le public ciblé. Le défi était de rendre le message de santé publique pertinent, intéressant et urgent auprès des jeunes. Au cours de cette éclosion, nous avons découvert que plusieurs jeunes adultes pensaient que leur protection vaccinale était complète et que les messages en lien avec la vaccination ne les concernaient pas. Ils ne se sentaient pas vulnérables face à la maladie ni ne percevaient l'urgence de la vaccination. Afin de s'attaquer à ce problème, la stratégie des réseaux sociaux s'est concentrée sur la partie «hipster» du public cible, soulignant les conséquences sociales de la maladie, comme manquer des activités sociales ou se sentir mis à l'écart. La réponse a été généralement positive, comparable à la réaction observée lors d'autres éclosions (12).

Plusieurs personnes atteintes et leurs contacts ont éprouvé des difficultés à trouver leurs dossiers de vaccination (39\% des cas). Encourager la vaccination plutôt qu'une analyse sérologique de l'immunité chez les personnes dont le dossier est inconnu est devenu un message important auprès des fournisseurs de soins de santé. Sans la présence d'un registre, il est difficile de connaître le nombre de personnes qui ont été vaccinées en réponse à l'éclosion; cependant, une mesure de substitution, le nombre de vaccins distribués aux fournisseurs de soins de santé, a montré une augmentation de la demande de vaccins antiourlien au plus fort de l'éclosion.

D'autres importantes éclosions ont été signalées en Amérique du Nord au cours des dernières années et la majorité d'entre elles se sont produites à l'école, au collège ou au sein d'équipes sportives. Un grand nombre ont été déclarées dans des populations qu'on disait bénéficier d'une couverture vaccinale complète $(13,14)$. Récemment, le Comité consultatif sur les pratiques d'immunisation aux États-Unis (Advisory Committee on Immunization Practices) a recommandé une troisième dose 
de vaccin antiourlien sur les lieux d'éclosions où la présence de personnes infectées ayant déjà reçu deux doses du vaccin est élevée (11). Dans le cas de l'éclosion de Toronto, presque le tiers des cas $(27 \%)$ représentaient des adultes complètement vaccinés.

Il est souvent difficile de déterminer la raison de la fin d'une éclosion. Dans le cas présent, il s'agissait d'une éclosion communautaire dans un grand centre urbain qui a duré 13 mois. II n'y a pas eu de transmission soutenue dans les écoles. Les messages de santé publique visant à modifier les comportements sociaux, comme échanger des ustensiles dans un bar ou au restaurant, peuvent avoir aussi eu leur importance. L'augmentation de la vaccination a possiblement joué un rôle dans la fin de l'éclosion. Bien que 12000 doses de vaccins supplémentaires aient été administrées sur une période de 6 mois au plus fort de l'éclosion comparativement à l'année suivante, il est difficile, sans registre, de déterminer l'importance de la cohorte de jeunes adultes vulnérables qui demeurent à Toronto.

\section{Limites}

La sous-déclaration de cas est probable pour plusieurs raisons : le fait que les cliniciens aient procédé à des examens inadéquats ou incomplets ou qu'ils n'en aient pas fait du tout; le fait que les patients légèrement atteints ou asymptomatiques soient moins susceptibles de consulter un médecin; et le fait que certaines personnes atteintes hésitaient à divulguer le nom de leurs contacts présentant des symptômes. Le statut d'immunisation était difficile à vérifier puisque les personnes atteintes et leurs contacts n'avaient souvent pas de dossiers de vaccination disponibles.

\section{Conclusion}

Parmi les cohortes vulnérables de jeunes adultes, les campagnes continues traditionnelles et sur les réseaux sociaux peuvent contribuer au contrôle des éclosions communautaires d'oreillons. Encourager la prise vaccinale est une bonne chose, mais, sans la présence d'un registre vaccinal, il est difficile d'évaluer la couverture vaccinale chez les adultes. Les cohortes vulnérables de jeunes adultes qui n'ont pas été immunisés de façon adéquate en raison des politiques de vaccinations mises en place par le passé sont un risque de futures éclosions. De plus, étant donné que près de $30 \%$ des cas concernaient des personnes considérées comme totalement immunisées par une double dose de vaccin contenant le virus des oreillons, deux doses pourraient ne pas offrir une protection complète.

\section{Déclaration des auteurs}

Tous les auteurs avaient accès aux données, ont contribué à la préparation et à la révision de l'article, et ont approuvé la version finale.

\section{Conflit d'intérêt}

Aucun.

\section{Remerciements}

Les auteurs souhaitent remercier les employés du Bureau de la santé publique de Toronto qui étaient membres de l'équipe de gestion de l'éclosion, l'équipe des maladies évitables par la vaccination et ceux et celles qui ont participé aux campagnes de communication et des réseaux sociaux, de même que nos collègues de la santé publique provinciale.

\section{Références}

1. Heymann DL, editor. Control of Communicable Diseases Manual. 20th edition. Washington (DC): American Association of Public Health; 2014.

2. Centers for Disease Control and Prevention. Epidemiology and Prevention of Vaccine-Preventable Diseases. Hamborsky J, Kroger A, Wolfe S, editors. 13th ed. Washington (DC): Public Health Foundation; 2015. 12 p. www.cdc.gov/ vaccines/pubs/pinkbook/index.html

3. L'Agence de la santé publique du Canada. Lignes directrices pour la prévention et le contrôle des éclosions d'oreillons au Canada. Relevé des maladies transmissibles au Canada 2010;36 Suppl:1-46. https://www.canada.ca/ content/dam/phac-aspc/migration/phac-aspc/publicat/ ccdr-rmtc/10pdf/36s1-fra.pdf DOI

4. Watson-Creed G, Saunders A, Scott J, Lowe L, Pettipas J, Hatchette TF. Two successive outbreaks of mumps in Nova Scotia among vaccinated adolescents and young adults. CMAJ 2006 Aug;175(5):483-8. www.cmaj.ca/ content/175/5/483 DOl PubMed

5. Deeks SL, Lim GH, Simpson MA, Gagné L, Gubbay J, Kristjanson E, Fung C, Crowcroft NS. An assessment of mumps vaccine effectiveness by dose during an outbreak in Canada. CMAJ 2011 Jun;183(9):1014-20. www.cmaj.ca/ content/early/2011/05/16/cmaj.101371 DOI PubMed

6. Toronto Public Health. Childhood Immunization Coverage in Toronto - Report from Medical Officer of Health, January 26, 2009. www.toronto.ca/legdocs $/ \mathrm{mmis} / 2009 / \mathrm{hl} / \mathrm{bgrd} /$ backgroundfile-18659.pdf

7. Ontario Agency for Health Protection and Promotion (Public Health Ontario). Immunization Coverage Report for School Pupils in Ontario: 2016-17 School Year. Toronto (ON): Queen's Printer for Ontario; 2018. www. publichealthontario. ca/en/eRepository/immunization-coverage-report-2016-17. pdf

8. Comité consultatif national de l'immunisation. Vaccin contre les oreillons: Guide canadien d'immunisation. Ottawa (ON): ASPC. https://www.canada.ca/fr/sante-publique/services/ publications/vie-saine/guide-canadien-immunisation-partie4-agents-immunisation-active/page-14-vaccin-oreillons.html

9. Ontario Public Health Standards, Infectious Diseases Protocol, Appendix A: Disease-Specific Chapters - Mumps. Revised January 2014. www.health.gov.on.ca/en/pro/ 
programs/publichealth/oph_standards/docs/mumps_ chapter.pdf

10. Gouvernement de I'Ontario, immunisation des élèves (Loi sur I'), L.R.O. 1990, chap. I.1. 1 septembre 2017. https://www. ontario.ca/fr/lois/loi/90i01

11. Marin M, Marlow M, Moore KL, Patel M. Recommendation of the Advisory Committee on Immunization Practices for Use of a Third Dose of Mumps Virus-Containing Vaccine in Persons at Increased Risk for Mumps During an Outbreak. MMWR Morb Mortal Wkly Rep 2018 Jan;67(1):33-8. www. cdc.gov/mmwr/volumes/67/wr/mm6701a7.htm DOI PubMed

12. Ross C, Shaw S, Marshall S, Stephen S, Bailey K, Cole R, et al. Incidence d'une campagne dans les médias sociaux ciblant les hommes ayant des relations sexuelles avec d'autres hommes pendant une éclosion de syphilis à
Winnipeg, Canada. Relevé des maladies transmissibles au Canada 2016;42-2:51-56. DOI PubMed

13. Bonwitt J, Kawakami V, Wharton A, Burke RM, Murthy N, Lee A, Dell B, Kay M, Duchin J, Hickman C, McNall RJ, Rota PA, Patel M, Lindquist S, DeBolt C, Routh J. Notes from the Field: Absence of Asymptomatic Mumps Virus Shedding Among Vaccinated College Students During a Mumps Outbreak - Washington, February-June 2017. MMWR Morb Mortal Wkly Rep 2017 Dec;66(47):1307-8. www.cdc.gov/ mmwr/volumes/66/wr/mm6647a5.htm DOI PubMed

14. Albertson JP, Clegg WJ, Reid HD, Arbise BS, Pryde J, Vaid A, Thompson-Brown R, Echols F. Mumps Outbreak at a University and Recommendation for a Third Dose of Measles-Mumps-Rubella Vaccine - Illinois, 2015-2016. MMWR Morb Mortal Wkly Rep 2016 Jul;65(29):731-4. www. cdc.gov/mmwr/volumes/65/wr/mm6529a2.htm DOI PubMed

\section{Annexe 1 :}

\section{Définitions des cas utilisées dans le cadre de l'éclosion d'oreillons de Toronto, du $1^{\text {er }}$ janvier 2017 au 16 février 2018}

\section{Confirmés}

Personne habitant ou visitant Toronto et présentant les caractéristiques suivantes :

1. une confirmation en laboratoire de l'infection pour une date de collecte de l'échantillon correspondant au 1 er janvier 2017 ou lui étant postérieure, accompagnée de symptômes cliniques compatibles avec les oreillons qui seraient apparus le 1 er janvier 2017 ou après

OU

2. des symptômes cliniques compatibles avec les oreillons qui seraient apparus le 1er janvier 2017 ou après chez une personne présentant un lien épidémiologique avec un cas confirmé en laboratoire associé à l'éclosion ET

3. une infection non associée à une exposition acquise en voyage

\section{Probables}

Personne habitant ou visitant Toronto et présentant les caractéristiques suivantes :

1. des symptômes cliniques compatibles avec les oreillons, dont les symptômes sont apparus le 1 er janvier 2017 ou après ET

2. un lien avec un site d'exposition connu lié à l'éclosion (absence de lien épidémiologique avec un cas confirmé en laboratoire ET

3. absence d'examen en laboratoire ou de confirmation du laboratoire (p. ex., les résultats de laboratoire sont en attente ou ne sont pas inclus dans la fenêtre de sensibilité des examens en laboratoire ET

4. une infection non associée à une exposition acquise en voyage 
Annexe 2 :

\section{Gestion des contacts dans le cadre de l'éclosion d'oreillons de Toronto, du $1^{\text {er }}$ janvier 2017 au 16 février 2018}

\section{Détermination des contacts}

On définissait un contact comme une personne à qui s'appliquait au moins un des critères suivants au cours de la période infectieuse (de sept jours avant à cinq jours après l'apparition des symptômes) :

1. contact à la maison avec une personne atteinte

2. fait de dormir dans la même pièce qu'une personne atteinte, y compris dans une salle commune ( $p$. ex. un dortoir) contact direct avec les sécrétions orales ou nasales d'une personne atteinte (p. ex. contact personnel, échanger des cigarettes, des boissons, des lunettes, des aliments, des cosmétiques comme des rouges à lèvres, s'embrasser sur la bouche)

3. enfant ou employé des garderies ou des établissements scolaires

4. travailleur en soins de santé ayant des interactions personnelles à moins d'un mètre d'un cas d'oreillons infectieux

5. fait de partager avec une personne atteinte les mêmes espaces intérieurs plus d'une heure par jour (p. ex. pendant des réunions sociales, comme des fêtes d'anniversaire ou la pratique du sport en équipe)

\section{Prise en charge des contacts}

Pour les contacts qui répondent aux critères ci-dessus, on a entrepris les actions suivantes :

1. les aviser de la possibilité d'une exposition aux oreillons et les informer sur la transmission de la maladie

2. déterminer le statut d'immunisation de l'ensemble des contacts; encourager la vaccination pour les personnes non immunisées ou sous-immunisées

3. noter les symptômes, le moment de leur apparition et leur gravité

4. considérer les contacts présentant des symptômes comme des cas possibles et procéder aux examens de confirmation

\section{Avis aux contacts}

Les services de santé publique ont avisé les contacts dans certaines situations, comme lorsqu'il s'agissait d'établissements de soins de santé ou d'écoles, si les ressources le permettaient. Cependant, pour un grand nombre de personnes atteintes au cours de l'éclosion, il a été impossible d'agir ainsi. C'est alors la personne atteinte qui a avisé ses contacts. Elle informait ses contacts, y compris au travail, de son exposition potentielle par messagerie électronique ou par téléphone et leur fournissait une lettre du Bureau de la santé publique de Toronto ainsi qu'une fiche d'information

\section{Contacts vulnérables}

Les personnes qui pourraient devoir être exclues des établissements de soins de santé ou des écoles sont les suivantes :

1. les personnes nées au Canada en 1970 ou après qui n'ont pas reçu deux doses de vaccins contenant le virus des oreillons (distancées d'au moins quatre semaines) le jour de leur premier anniversaire ou après

2. les personnes sans antécédents d'oreillons confirmés en laboratoire

3. les personnes dont l'immunité contre les oreillons n'est pas documentée 